\title{
Downregulation of miR-1184 serves as a diagnostic biomarker in neonatal sepsis and regulates LPS-induced inflammatory response by inhibiting IL-16 in monocytes
}

\author{
DAN WANG $^{*}$ and LINA HAN ${ }^{*}$ \\ Neonatology Department, Weifang People's Hospital, Weifang, Shandong 261041, P.R. China
}

Received June 3, 2020; Accepted December 18, 2020

DOI: $10.3892 /$ etm.2021.9781

\begin{abstract}
Neonatal sepsis (NS) remains a global problem. In the present study, abnormal expression of microRNA-1184 (miR-1184) was detected in neonates with NS and it was endeavored to investigate the diagnostic value of miR-1184, as well as its regulatory role in lipopolysaccharide (LPS)-induced inflammatory response in vitro. Furthermore, the correlation between interleukin-16 (IL-16) and miR-1184 was investigated to elucidate the pathological mechanisms of NS development. Reverse transcription-quantitative PCR was used to detect the expression of miR-1184. Receiver operating characteristic curve analysis was performed to evaluate the diagnostic value of miR-1184 in NS. Furthermore, a sepsis cell model was established by using LPS-induced monocytes to explore the effect of miR-1184 on the inflammatory response. The levels of inflammatory cytokines were determined by ELISA. A luciferase reporter assay was used to investigate the direct targeting interaction between miR-1184 and IL-16. The results indicated that the serum levels of miR-1184 in neonates with sepsis were decreased and miR-1184 had a high diagnostic value when differentiating NS from respiratory conditions in neonates. In vitro, the expression of miR-1184 in monocytes was inhibited by LPS and overexpression of miR-1184 reversed the effect of LPS to stimulate the inflammatory response. IL-16 was demonstrated to be a target of miR-1184 and a negative correlation between them was identified in patients with NS. The inflammatory response inhibited by miR-1184 mimics was enhanced by overexpression of IL-16 in LPS-induced monocytes. In conclusion, decreased levels of serum miR-1184 may be a potential diagnostic biomarker for NS. In addition, miR-1184 inhibited the LPS-induced
\end{abstract}

Correspondence to: Dr Lina Han, Neonatology Department, Weifang People's Hospital, 151 Guangwen Street, Kuiwen, Weifang, Shandong 261041, P.R. China

E-mail: superli1026@163.com

*Contributed equally

Key words: microRNA-1184, neonatal sepsis, diagnosis, inflammation, monocyte, interleukin-16 inflammatory response by targeting IL-16 in monocytes, suggesting that the miR-1184/IL-16 axis may be a potential therapeutic target for NS.

\section{Introduction}

Sepsis is a systemic inflammatory response syndrome in which pathogenic microorganisms invade the circulatory system, grow and multiply to produce endotoxins and exotoxins, and potentially induce injury to multiple organs (1). In newborns, the source of the pathogen may be an in utero infection, maternal flora infection or postpartum infection from the hospital or community (2). Neonatal sepsis (NS) is a leading cause of morbidity and mortality in newborns (3), and it may be categorized as early-onset NS (EOS) or late-onset NS (LOS) (4). The morbidity and mortality of NS have been reduced due to progress made in neonatal care (5). However, there have been minimal advances in the specific clinical management and accuracy of diagnostic testing options over the last several decades (6). Numerous sepsis biomarkers have been evaluated for their potential in early detection of NS, but to date, no ideal biomarker has met all of the basic criteria for being an ideal indicator (7). The most commonly used biomarkers are C-reactive protein (CRP) and procalcitonin (PCT), but both of them have demonstrated varying sensitivity, specificity and positive and negative predictive values in different studies (7). Blood culture has been considered as a gold standard method, but the identification of EOS is complicated by a high rate of false-negative results (8).

MicroRNAs (miRNAs/miRs) are a class of small ( 22 nucleotides), non-coding, single-stranded RNAs that regulate gene expression by binding the 3'-untranslated region (3'-UTR) of target mRNAs for mRNA degradation or translational suppression (9). They have been indicated to be frequently dysregulated in cancers and serve as attractive targets for prognostication and therapeutic applications (10). miRNAs have been used in fingerprint diagnosis of sepsis and have been identified as potential biomarkers of sepsis (11). In the pathogenesis of sepsis, certain miRNAs, such as miR-125b and miR-142-3p, have been demonstrated to regulate inflammation $(12,13)$. In addition, Chen et al (14) performed miRNA expression profiling using a microarray and determined that altered miRNAs (including miR-1184) may modulate the immune response during NS in a way that 
represses the inflammatory response. However, the association between miR-1184 and NS remains to be fully elucidated. Interleukin-16 (IL-16) is a cytokine associated with various inflammatory processes (15) and has been documented to facilitate the production of pro-inflammatory cytokines in monocytes (16). Studies have reported that IL-16 may be used as a target for certain miRNAs in cancer $(17,18)$. Furthermore, the study by Chen et al (14) used a protein chip and preliminarily determined that downregulation of miR-1184 in patients with NS was associated with increased protein levels of IL-16. Lipopolysaccharide (LPS) has been widely used to induce inflammatory responses that mimic the pathological processes of inflammation in human inflammatory diseases, including sepsis (19). However, the clinical value and biological function of the aberrant miR-1184 in NS remain to be fully elucidated.

The present study sought to explore the diagnostic value of serum miR-1184 by comparing the serum levels of miR-1184 between subjects with NS and healthy newborns. The effect of miR-1184 on the LPS-induced inflammatory response was analyzed in monocytes. The current study simultaneously upregulated miR-1184 and IL-16 levels in the cell model, then determined whether IL-16 was involved in the regulation of miR-1184 during the inflammatory response by detecting changes in inflammatory factor levels. Therefore, the present study may provide novel insight into the regulation of inflammation in the pathogenesis of NS.

\section{Materials and methods}

Patients and blood sample collection. The experimental protocols were approved by the Ethics Committee of Weifang People's Hospital (Weifang, China) and the parents of each neonate had provided written informed consent. Blood samples were collected from 72 neonates with NS (including 6 cases of EOS and 66 cases of LOS) and 56 neonates with respiratory infection or pneumonia who visited Weifang People's Hospital between April 2015 and December 2018. The latter were selected as controls as it is critical to differentiate between sepsis and respiratory infection to provide more effective treatment. Serum was isolated from the blood of all participants by centrifugation and stored at $-80^{\circ} \mathrm{C}$ for further use. None of the neonates had received any therapy prior to blood collection. The patients with NS were diagnosed according to the criteria established at the 2003 Kunming Neonatal Sepsis Definitions Conference (20) and the neonates with respiratory infection or pneumonia served as controls without any symptoms or signs of sepsis. The inclusion criteria for patients with NS were as follows: i) Term birth; ii) no antibiotic treatment prior to sample collection; iii) no inflammation or antibiotic therapy prior to delivery; iv) no congenital malformations; v) Apgar score of $>6$ at $5 \mathrm{~min}$. The diagnosis of NS mainly relied on the clinical manifestations and the detection of blood pathogens. The clinicopathological characteristics of the neonates included are listed in Table I.

Monocyte culture and LPS induction. Monocytes were collected from blood samples of subjects with NS according to a previously described method (21). In brief, the blood samples were settled with 4.5\% dextran 500 (1:5; Amersham Biosciences) to separate leukocytes from red blood cells.
The monocytes were then obtained using density gradient centrifugation and the cell purity was confirmed by FACSCalibur flow cytometry (BD Biosciences) based on the detection of the specific cell markers CD14 (cat. no. 562691; 1:20; BD Biosciences) and CD15 (cat. no. 560827; 1:20; BD Biosciences). The extracted monocytes were cultured in RPMI-1640 medium supplemented with 10\% FBS (both from Gibco; Thermo Fisher Scientific, Inc.) in a humidified atmosphere with $5 \% \mathrm{CO}_{2}$ at $37^{\circ} \mathrm{C}$. The monocytes were cultured for 7 days for further use. To explore the effects of miR-1184 on LPS-induced inflammatory response, the monocytes were stimulated using LPS (Sigma-Aldrich; Merck KGaA) for $4 \mathrm{~h}$. All of the experiments were performed at least 3 times.

Cell transfection. miR-1184 mimics (5'-CCUGCAGCGACU UGAUGGCUUCC-3') and mimics negative control (NC) (5'-UUCUCCGAACGUGUCACGU-3'), which were obtained from GenePharma, were used for cell transfection to regulate the expression of miR-1184 in monocytes. IL-16 overexpression vectors pcDNA3.1-IL-16 were constructed to upregulate IL-16 expression in monocytes and pcDNA3.1 empty vector (Invitrogen; Thermo Fisher Scientific, Inc.) was used as a control for pcDNA3.1-IL-16 (Shanghai GenePharma Co., Ltd.). The above vectors were separately transfected into monocytes using Lipofectamine 2000 (Thermo Fisher Scientific, Inc.) according to the manufacturer's protocols. Cells were collected after transfection for $48 \mathrm{~h}$ at $37^{\circ} \mathrm{C}$ and used for the subsequent analyses.

$R N A$ extraction and reverse transcription-quantitative $P C R$ $(R T-q P C R)$. The collected blood was centrifuged to isolate the serum samples. TRIzol ${ }^{\circledR}$ reagent (Invitrogen; Thermo Fisher Scientific, Inc.) was used to extract total RNA from serum or monocyte samples, including miRNA. A NanoDrop 2000 (Thermo Fisher Scientific, Inc.) was used to evaluate the purity and concentration of the RNA. The complementary DNA was then synthesized from the obtained RNA by RT using a PrimeScript RT reagent kit (Takara Bio, Inc.) according to the manufacturer's protocol.

The serum levels of miR-1184 and mRNA serum/monocyte levels of IL-16 were measured using RT-qPCR, which was performed using a SYBR Green I Master Mix kit (Invitrogen; Thermo Fisher Scientific, Inc.) and a 7300 Real-Time PCR System (Applied Biosystems; Thermo Fisher Scientific, Inc.). All of the procedures were performed according to the manufacturer's instructions. U6 was used as an endogenous control for miR-1184 and GAPDH was used as an internal control for IL-16. The primers used for analysis were as follows: miR-1184 forward, 5'-GCCGAGCCTGCA GCGACTTG-3' and reverse, 5'-CTCAACTGGTGTCGT GGA-3'; IL-16 forward, 5'-GGAATCGTGCTTCAGACC CA-3' and reverse, 5'-CTCTGGGCTCCTTTGTCAGG-3'; U6 forward, 5'-CTCGCTTCGGCAGCACA-3' and reverse, 5'-AA CGCTTCACGAATTTGCGT-3'; GAPDH forward, 5'-GCT CCCTCTTTCTTTGCAGC-3' and reverse, 5'-GTTGTC ATGGATGACCTTGGC-3'. The relative expression values were calculated using the $2^{-\Delta \Delta \mathrm{Cq}}$ method (22).

ELISA. To evaluate the status regarding the inflammatory response, the levels of pro-inflammatory cytokines, including 
Table I. Baseline characteristics of the study population.

\begin{tabular}{lccr}
\hline Feature & Controls $(\mathrm{n}=56)$ & NS (n=72) & P-value \\
\hline Age (days) & $12.8 \pm 4.6$ & $12.8 \pm 4.2$ & 0.988 \\
Sex (female/male) & $24 / 32$ & $33 / 39$ & 0.737 \\
Body weight $(\mathrm{kg})$ & $3.7 \pm 0.3$ & $3.6 \pm 0.3$ & 0.225 \\
WBC $\left(\mathrm{x} 10^{9} / \mathrm{l}\right)$ & $10.7(8.1-13.5)$ & $11.7(8.2-14.7)$ & 0.130 \\
CRP $(\mathrm{mg} / \mathrm{l})$ & $11.8(9.7-14.1)$ & $13.9(9.7-17.4)$ & 0.016 \\
PCT $(\mathrm{ng} / \mathrm{ml})$ & $2.0(1.3-2.8)$ & $4.5(2.8-5.8)$ & $<0.001$
\end{tabular}

Values are expressed as the mean \pm standard deviation, $\mathrm{n}$ or median (interquartile range). Age and weight differences between the two groups were analyzed using Student's t-test; the $\chi^{2}$ was used to compare the difference in gender; the Mann-Whitney U-test was used to analyze differences in WBC, CRP and PCT. NS, neonatal sepsis; WBC, white blood cells; CRP, C-reactive protein; PCT, procalcitonin.

IL-1 $\beta$, IL-6 and TNF- $\alpha$, in both serum samples and monocytes were examined by ELISA. For cell lysis, $100 \mu 1 \mathrm{RIPA}$ lysis buffer (0.5 M Tris- $\mathrm{HCl}, \mathrm{pH} 7.4,1.5 \mathrm{M} \mathrm{NaCl}, 2.5 \%$ deoxycholic acid, $10 \%$ NP-40 and 10 mM EDTA) was added to per $1 \times 10^{6}$ cells. The serum and cell lysate were examined using ELISA kits for IL-1 $\beta$ (cat. no. 557953), IL-6 (cat. no. 555220) and TNF- $\alpha$ (cat. no. 555212; all from BD Biosciences) according to the manufacturer's protocols. The absorbance value at $450 \mathrm{~nm}$ was detected with a microplate reader (Bio-Rad Laboratories, Inc.). The concentration of the cytokines in cell lysate was quantified using standard curves.

Luciferase reporter assay. The putative binding site of miR-1184 at the 3'-UTR of IL-16 was predicted using miRanda (http://www.microrna.org/microrna/home.do). To confirm whether there was a direct interaction between miR-1184 and IL-16, a luciferase reporter assay was performed. The wild-type (WT) 3'-UTR containing the binding site for miR-1184 was amplified by PCR, and the mutant-type (MUT) 3'-UTR was generated using a QuickMutation kit (Beyotime Institute of Biotechnology). The 3'-UTR sequences were inserted in the pGL-control vector (Promega, Corp.). The vectors constructed were separately transfected into monocytes together with either miR-1184 mimics or mimics NC using Lipofectamine 3000 (Thermo Fisher Scientific, Inc.) according to the manufacturer's protocols. After $48 \mathrm{~h}$ of transfection, the activity of luciferase was evaluated using a Dual-Luciferase Reporter Assay System (Promega, Corp.) and normalized to Renilla luciferase activity.

Statistical analysis. All statistical analyses were performed by using SPSS 21.0 software (IBM Corp.) and GraphPad Prism 7.0 software (GraphPad Software, Inc.). Values are expressed as the mean \pm standard deviation, number or median (interquartile range). Differences between two groups were analyzed using Student's t-test, the $\chi^{2}$ or the Mann-Whitney U-test. One-way ANOVA followed by Tukey's test were used to compare differences among multiple groups. A receiver operating characteristic (ROC) curve was plotted to evaluate the ability of miR-1184 to differentiate between patients with NS and neonates with respiratory infection/pneumonia. Pearson's correlation analysis was performed to determine the correlation coefficient. $\mathrm{P}<0.05$ was considered to indicate statistical significance.

\section{Results}

Baseline characteristics of patients with NS and controls. In the present study, a total of 72 neonates with NS and 56 newborns with respiratory infection/pneumonia as controls were enrolled. Their baseline characteristics are summarized in Table I. The neonates with NS included 33 females and 39 males with an average age of $12.8 \pm 4.2$ days. The controls included 24 females and 32 males with an average age of $12.8 \pm 4.6$ days. There were no significant differences in age, gender, body weight and white blood cell (WBC) count (all $\mathrm{P}>0.05$ ) between the cases of NS and controls, whereas the patients with NS had markedly higher levels of CRP and PCT than the controls (both $\mathrm{P}<0.05$ ).

Serum levels of miR-1184 and IL-16 and their correlation in patients with NS. The serum levels of miR-1184 and IL-16 mRNA in neonates with sepsis and controls were measured using RT-qPCR. As presented in Fig. 1A and B, the neonates with NS had significantly lower miR-1184 levels and higher IL-16 mRNA levels than those in the control group $(\mathrm{P}<0.001$ for each). Furthermore, a negative correlation between the serum levels of miR-1184 and IL-16 was observed in neonates with NS (r=-0.824, P<0.001; Fig. 1C).

Correlation between miR-1184 and inflammatory response in patients with NS. The inflammatory response in patients with NS was evaluated by measuring the levels of serum pro-inflammatory cytokines, including IL-1 $\beta$, IL- 6 and TNF- $\alpha$. The results presented in Table II and Fig. 2 indicated that the serum levels of miR-1184 were negatively correlated with the serum levels of IL-1 $\beta$, IL- 6 and TNF- $\alpha$, which suggested a potential relationship between miR-1184 and the inflammation in the progression of NS.

Diagnostic value of miR-1184 for NS. Due to the significant dysregulation of miR-1184 in the serum of patients with NS, the diagnostic significance of serum miR-1184 was assessed in the present study. The ROC curves for CRP (Fig. 3A) and PCT (Fig. 3B), which are two widely used markers to diag- 
A

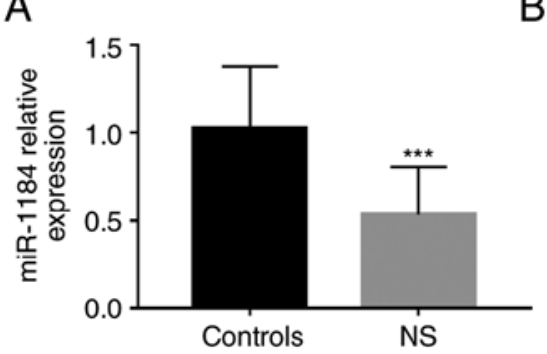

B

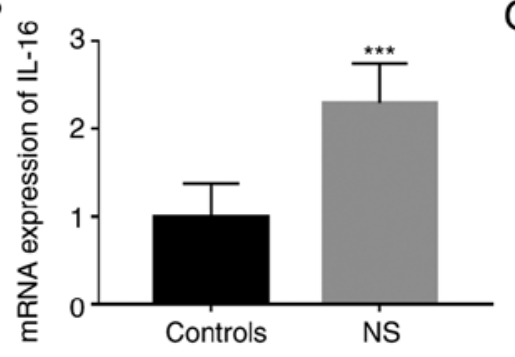

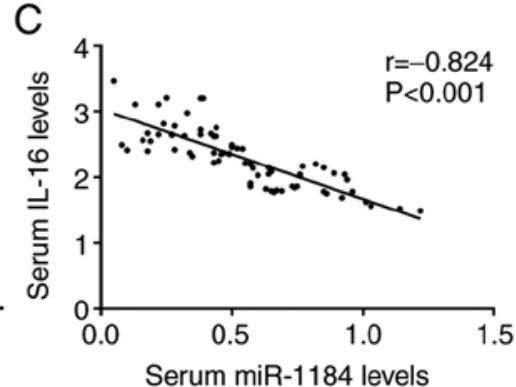

Figure 1. Serum miR-1184 levels, mRNA levels of IL-16 and their correlation in subjects with NS. (A) Serum miR-1184 levels in patients with NS were decreased compared with those in the controls. (B) Relative mRNA expression of serum IL-16 was higher in patients with NS than that in controls. (C) Serum miR-1184 expression levels were negatively correlated with IL-16 levels in patients with NS. The number of patients with NS was 72 and that of the controls was $56 .{ }^{* * *} \mathrm{P}<0.001$ vs. control. NS, neonatal sepsis; miR, microRNA; IL, interleukin.

A

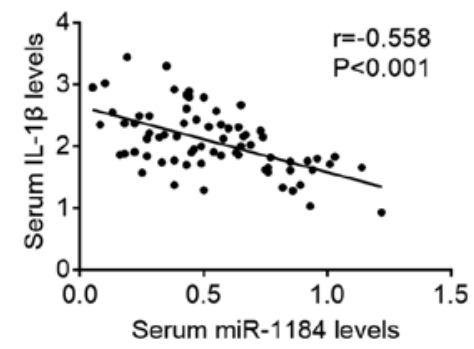

B

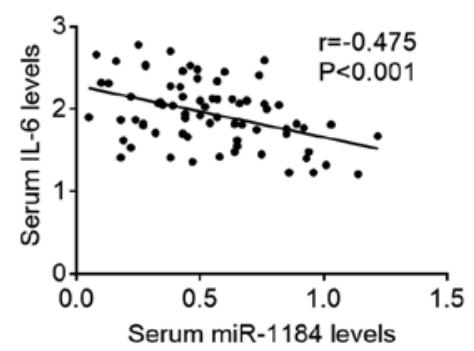

C

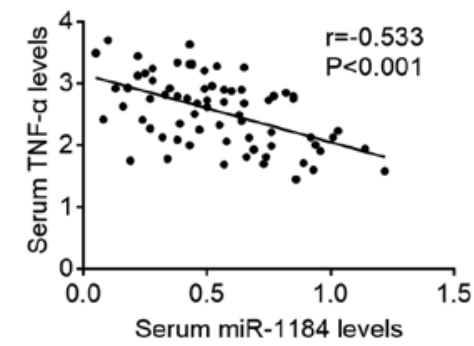

Figure 2. Correlation between serum miR-1184 levels and serum pro-inflammatory cytokines in neonates with sepsis. Serum miR-1184 expression levels were negatively correlated with serum (A) IL-1 $\beta$, (B) IL-6 and (C) TNF- $\alpha$ levels in neonates with sepsis. The number of patients was 72 . miR, microRNA; IL, interleukin; TNF, tumor necrosis factor.

nose sepsis, were first constructed. The area under the curve (AUC) for CRP was 0.625 with a sensitivity of $45.83 \%$ and a specificity of $83.93 \%$ at a cutoff value of 15.370 . The AUC for PCT was 0.824 with a sensitivity of $69.44 \%$ and a specificity of $92.86 \%$ at a cutoff value of 3.375 . The ROC curve for the levels of miR-1184 is presented in Fig. 3C; the AUC was 0.861 with a sensitivity and specificity of 80.56 and $83.93 \%$, respectively, at a cutoff value of 1.307 .

miR-1184 ameliorates LPS-induced inflammation in monocytes. In the present study, monocytes were induced with LPS to establish an in vitro model of sepsis with active inflammation. Similar to the results in neonates with sepsis, the expression levels of miR-1184 were also downregulated in cells after LPS induction compared with those in untreated cells $(\mathrm{P}<0.001$; Fig. 4A). Following transfection of the miR-1184 mimics, the expression levels of miR-1184 were confirmed to be significantly upregulated in the monocytes $(\mathrm{P}<0.001$; Fig. 4A). Furthermore, overexpression of miR-1184 in monocytes reversed the effect of LPS to increase the levels of IL-1 $\beta$, IL-6 and TNF- $\alpha$ (all P<0.001; Fig. 4B), which meant that miR-1184 was able to weaken the LPS-induced inflammatory response.

miR-1184 directly regulates $I L-16$ expression in monocytes. In order to further confirm the direct interaction between miR-1184 and IL-16, a luciferase reporter assay was performed. A binding site for miR-1184 was identified in the 3'-UTR of IL-16 (Fig. 5A). A subsequent luciferase reporter
Table II. Correlation between serum miR-1184 levels and pro-inflammatory cytokines in neonates with sepsis.

\begin{tabular}{llr}
\hline & \multicolumn{2}{c}{ miR-1184 } \\
\cline { 2 - 3 } Cytokine & r-value & P-value \\
\hline IL-1 $\beta$ & -0.558 & $<0.001$ \\
IL-6 & -0.475 & $<0.001$ \\
TNF- $\alpha$ & -0.533 & $<0.001$ \\
\hline
\end{tabular}

miR, microRNA.

assay indicated that miR-1184 mimics markedly inhibited the relative luciferase activity in the WT group $(\mathrm{P}<0.05)$, while there was no change in the MUT group (Fig. 5B). In addition, the LPS-induced elevation of IL-16 mRNA expression in monocytes was inhibited by overexpression of miR-1184 $(\mathrm{P}<0.01$; Fig. 5C). These data suggested that miR-1184 directly regulates IL-16 in monocytes.

Effect of miR-1184/IL-16 on LPS-induced inflammatory response in monocytes. To verify whether IL-16 mediated the regulation of miR-1184 on LPS-induced inflammation, IL-16, which was inhibited by miR-1184 mimics, was overexpressed using pcDNA3.1-IL-16 ( $\mathrm{P}<0.01$; Fig. 6A). Regarding the inflammatory responses in LPS-induced 

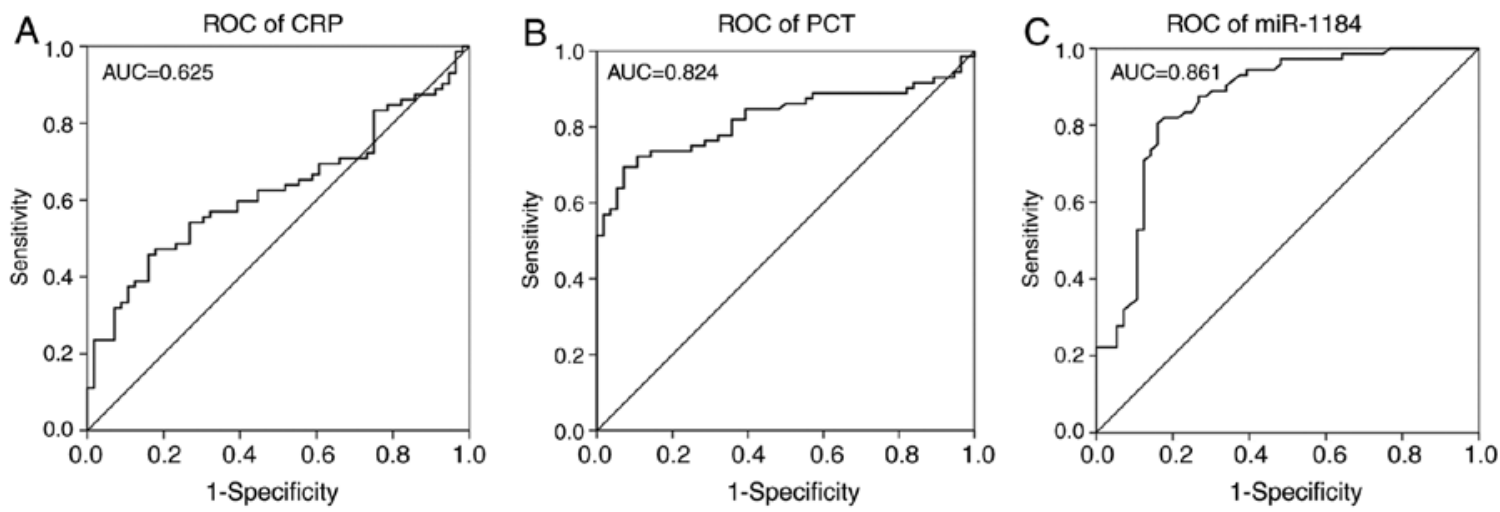

Figure 3. ROC curves for the detection of sepsis in neonates based on serum indicators. ROC curves for (A) CRP (AUC was 0.625 at the optimal cutoff value of 15.370), (B) PCT (AUC was 0.824 at the optimal cutoff value of 3.375) and (C) miR-1184 (AUC was 0.861 at the optimal cutoff value of 1.307). The number of patients was 72. ROC, receiver operating characteristic; AUC, area under the curve; CRP, C-reactive protein; PCT, procalcitonin; miR, microRNA.
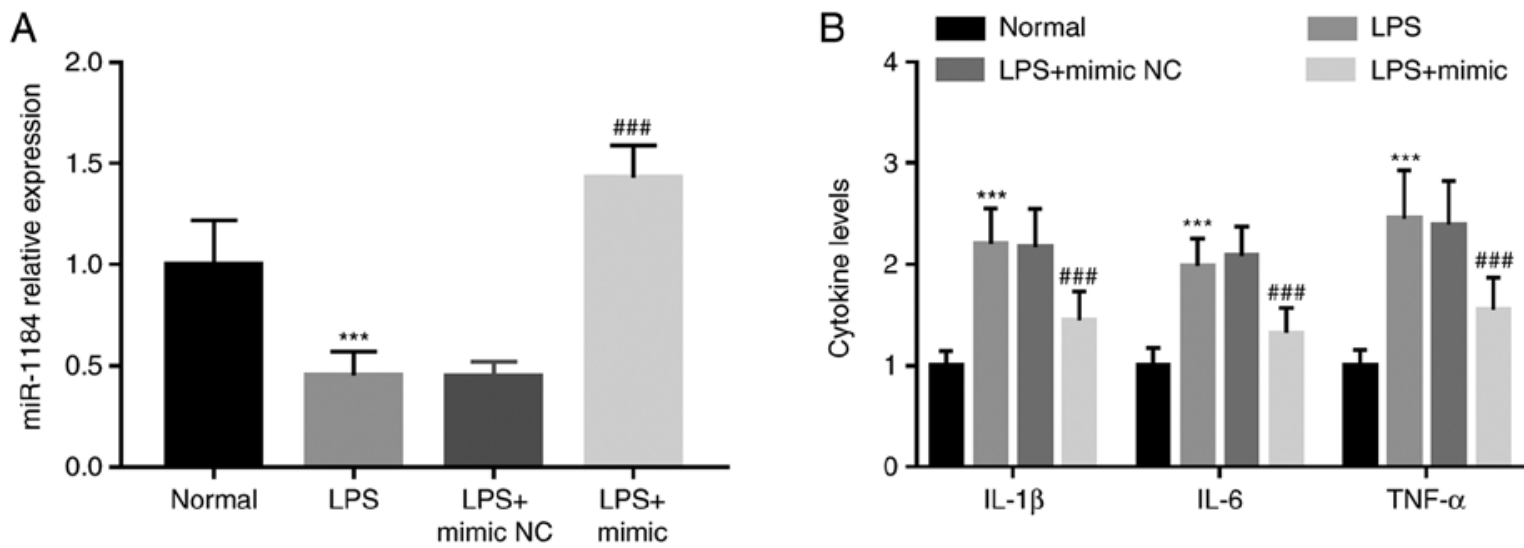

Figure 4. Regulatory effect of miR-1184 on LPS-induced inflammatory responses in monocytes. (A) The expression of miR-1184 was inhibited by LPS and was decreased by miR-1184 mimics in monocytes. (B) Overexpression of miR-1184 reversed the promoting effect of LPS on the levels of the inflammatory cytokines IL-1 $\beta$, IL-6 and TNF- $\alpha$. All experiments were performed at least three times. ${ }^{* * *} \mathrm{P}<0.001$ vs. Normal; ${ }^{\# \# \#} \mathrm{P}<0.001$ vs. LPS. LPS, lipopolysaccharide; miR, microRNA; NC, negative control; IL, interleukin.

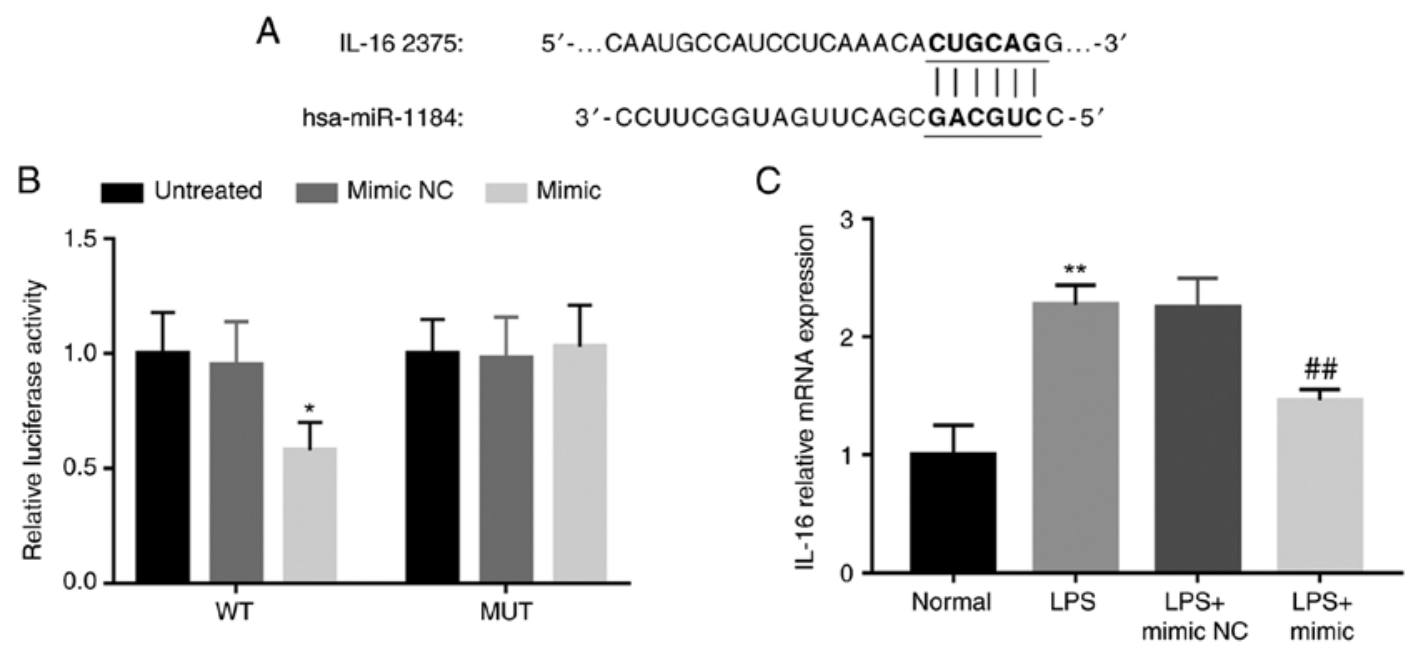

Figure 5. miR-1184 directly inhibits the level of IL-16 in monocytes. (A) Putative complementary sequence of miR-1184 in the 3'-untranslated region of IL-16. (B) Luciferase activity was inhibited by overexpression of miR-1184 in the WT group. (C) The increase of IL-16 induced by LPS was inhibited by overexpression of miR-1184. All experiments were performed at least three times. ${ }^{*} \mathrm{P}<0.05,{ }^{* * *} \mathrm{P}<0.01$ vs. untreated or Normal; ${ }^{\# \#} \mathrm{P}<0.01$ vs. LPS. LPS, lipopolysaccharide; miR, microRNA; NC, negative control; IL, interleukin; MUT, mutant type; WT, wild-type; hsa, Homo sapiens.

monocytes, the results suggested that the inhibition of IL-1 $\beta$, IL-6 and TNF- $\alpha$ following transfection with miR-1184 mimics was abolished by simultaneous overexpression of IL-16 ( $\mathrm{P}<0.001$; Fig. 6B). 

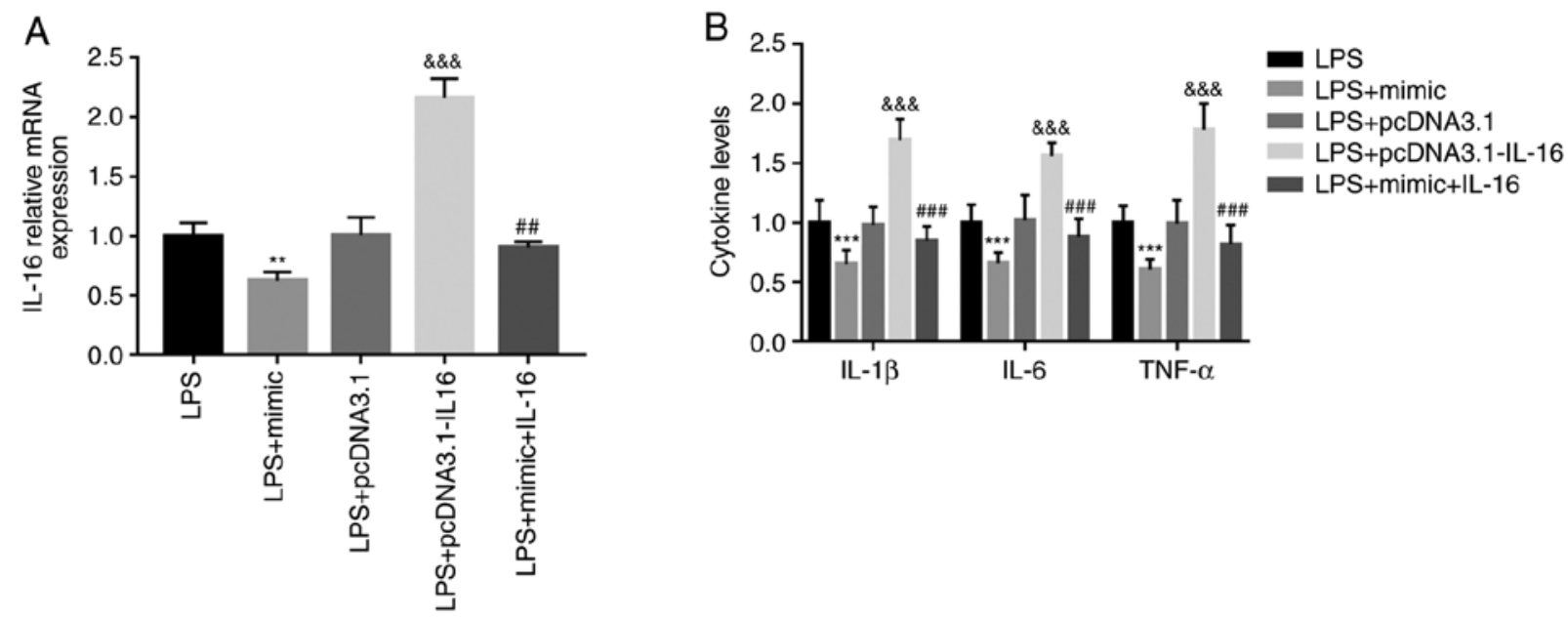

Figure 6. IL-16 mediates the inhibitory effect of miR-1184 on LPS-induced inflammatory responses. (A) The inhibition of IL-16 expression following overexpression of miR-1184 was abolished in monocytes after transfection with pcDNA3.1-IL-16. (B) Inhibition of inflammatory cytokine levels, including IL-1 $\beta$, IL-6 and TNF- $\alpha$ following overexpression of miR-1184 was reversed by the increase of IL-16 in monocytes. All experiments were performed at least three times. ${ }^{* *} \mathrm{P}<0.01,{ }^{* * *} \mathrm{P}<0.001$ vs. LPS; ${ }^{\& \& \&} \mathrm{P}<0.001$ vs. LPS+pcDNA3.1; ${ }^{\# \#} \mathrm{P}<0.01,{ }^{\# \# \#} \mathrm{P}<0.001$ vs. LPS+mimics. LPS, lipopolysaccharide; miR, microRNA; $\mathrm{NC}$, negative control; IL, interleukin.

\section{Discussion}

Numerous studies have indicated that miRNAs have pivotal roles in the development of various human diseases, including cancer (23), neurodegenerative diseases (24) and metabolic diseases (25). There are various functional miRNAs that are associated with inflammatory disease progression by regulating the inflammatory response, including NS. For instance, miR-223 is an anti-inflammatory miRNA that is mainly expressed in myeloid cells (26). miR-144 was observed to induce autophagy and inflammatory responses in microglia after cerebral hemorrhage by regulating mTOR (27). Overexpression of miR-300 enhanced autophagy by targeting nicotinamide phosphoribosyltransferase to reduce the inflammatory response (28). miR-26a was reported to participate in the pathogenesis of NS by targeting its downstream IL-16 (29). These studies highlight the key roles of miRNAs in the pathogenesis of inflammatory diseases, including NS, by regulating inflammatory responses. In the present study, subjects with NS and neonates with respiratory tract infection or pneumonia were used as controls, but healthy neonates were not recruited. Consistent with the results of a previous study by Chen et al (14), who reported that miR-1184 may directly regulate IL-16 and was downregulated in neonates with sepsis compared with uninfected neonates. The results of the present study revealed that miR-1184 expression was decreased in neonates with sepsis compared with neonates exhibiting respiratory tract infection or pneumonia, and decreased in LPS-induced monocytes compared with normal cells. Therefore, the abnormal expression of miR-1184 in NS suggested that miR-1184 may be involved in the development of NS.

Accurate diagnosis is the first and most important prerequisite for the efficient treatment of diseases. To improve the treatment of infectious diseases in neonates, it is of value to distinguish cases of NS from neonates with pneumonia/respiratory tract infection (30). CRP and PCT were established as biomarkers for NS and are widely used for this purpose, but elevated CRP and PCT may also be detected in certain infectious and inflammatory diseases other than NS, such as pneumonia (31) and respiratory tract infection (32); thus, their application specificity is limited. At present, the existing diagnostic methods for NS are limited due to their poor sensitivity and specificity. Thus, novel biomarkers with high sensitivity and specificity are needed to improve the diagnosis of NS. miRNAs are stable and can be easily detected from body fluids, such as serum and tears, meaning they may be detected as biomarkers in biological fluids and archival tissues $(33,34)$. Plasma miR-1290 was reported as a novel and specific biomarker for early diagnosis of necrotizing enterocolitis (35). miR-15a and miR-16 have been evaluated in blood samples from patients with NS and had were indicated to be diagnostic and prognostic biomarkers for NS (11). In the present study, no significant differences were observed in age, sex, body weight and WBC between the controls and subjects with NS. However, the cases with NS had significantly higher levels of CRP and PCT than the controls, suggesting that the levels of CRP and PCT may be able to screen NS cases from the neonates with pneumonia/respiratory tract infection. Thus, ROC curve analysis for CRP and PCT to distinguish NS from respiratory conditions in neonates was performed. Given the significant downregulation of miR-1184 in serum samples of patients with NS, a ROC curve for miR-1184 was also constructed to compare its diagnostic value with that of CRP and PCT. The analysis indicated that abnormal expression of miR-1184, which may be used as a biomarker for the diagnosis of NS, had a relatively high diagnostic accuracy in newborns with considerable sensitivity and specificity, and had a better diagnostic performance compared with CRP and PCT. The present study may provide novel and effective diagnostic biomarkers for NS. Previous studies have indicated that biomarkers used for EOS and LOS may not be the same. For instance, a previous study reported differentially expressed plasma soluble CD14 subtypes between EOS and LOS, and emphasized the importance to notice differences when treating EOS and LOS (36). Furthermore, miR-23b expres- 
sion, which may serve as a good marker, was upregulated in EOS but downregulated in LOS, and its expression was altered during different neonatal periods (37). In the present study, only 6 cases of EOS were included in the NS group, which was not sufficient to distinguish EOS from LOS for analysis. Considering the potential differences in the progression of EOS and LOS, future studies by our group will expand the sample size and perform analyses in EOS and LOS separately.

NS is characterized by an inflammatory response. To mimic the activated inflammation in sepsis, LPS-induced monocytes have been widely used to investigate the molecules involved in the inflammation that is part of the pathogenesis of sepsis (38). Zhang et al (39) suggested that the circulating levels of miR-23b were decreased in patients with sepsis and were able to inhibit the LPS-induced inflammatory response in monocytes. Overexpression of miR-181a inhibited the LPS-induced inflammatory response at least partially by targeting Toll-like receptor 4 (30). The present study determined that the expression of miR-1184 was also downregulated in LPS-induced monocytes and overexpression of miR-1184 reversed the promoting effect of LPS on the inflammatory response in monocytes. These results suggested that miR-1184 may be involved in NS progression by regulating the inflammatory response and may be used as a therapeutic target.

The C-terminal fragment IL-16C is a bioactive protein that is a secreted pro-inflammatory cytokine (40). IL-16 has been reported to be upregulated in patients with NS (41). A previous study suggested that IL-16 was a target of miR-1184 and was inhibited by miR-1184 (14). In the present study, the results of a luciferase reporter assay and the relative mRNA expression data of IL-16 in monocytes indicated that miR-1184 directly regulates the expression of IL-16 in monocytes. Furthermore, the present study simultaneously upregulated miR-1184 and IL-16 levels in LPS-induced monocytes to determine whether IL-16 was involved in the regulation of miR-1184 during the inflammatory response by detecting the changes in inflammatory factor levels. The results indicated that in monocytes co-transfected with miR-1184 and IL-16 overexpression vectors, the inhibitory effect of miR-1184 mimics on inflammation was abolished by overexpression of IL-16. In addition, serum miR-1184 in patients with NS was negatively correlated with IL-16. These results suggested that the inhibitory effect of miR-1184 on inflammation in NS may be achieved by inhibiting IL-16. IL-16 has been indicated to facilitate the production of pro-inflammatory cytokines in monocytes, such as IL-1 $\beta$, IL-6, IL-15 and TNF- $\alpha$ (16). In addition, Huang et al (42) reported that IL-16 regulated IL-10, IL-1a and IL-6 expression and served as a mediator of miR-145-3p to regulate macrophage polarization. Thus, IL-16 may also act as an important molecule in the pathogenesis of NS and the miR-1184/IL-16 axis may be a novel therapeutic target for the treatment of NS.

In conclusion, the present study indicated that downregulated miR-1184 in the serum of neonates with sepsis may be closely linked to NS-related inflammation and may be used as a candidate biomarker for the diagnosis of NS. Overexpression of miR-1184 in monocytes may improve the LPS-induced inflammatory response by targeting IL-16, suggesting that the miR-1184/IL-16 axis may provide a novel therapeutic target for the treatment of NS. A limitation of the present study is the use of only in vitro experiments to evaluate the regulatory effects of miR-1184 on NS-related inflammation, with no in vivo animal experiments (43), which may be one of the limitations of the present study. In addition, the sample size was relatively small, which may limit the accuracy of the clinical study data and the understanding of the potential role of the miR-1184/IL-16 axis in cases with EOS or LOS individually. Thus, further investigations in a large study cohort are necessary.

\section{Acknowledgements}

Not applicable.

\section{Funding}

No funding received.

\section{Availability of data and materials}

The datasets used and/or analyzed during the current study are available from the corresponding author on reasonable request.

\section{Authors' contributions}

DW and LH designed and conceived the study, conducted clinical and cellular experiments, analyzed the data, wrote the manuscript, and confirmed the authenticity of the raw data. All authors read and approved the final manuscript.

\section{Ethics approval and consent to participate}

Written informed consent was obtained from the parents of each patient and the experimental procedures were approved by the Ethics Committee of Weifang People's Hospital (Weifang, China).

\section{Patient consent for publication}

Not applicable.

\section{Competing interests}

The authors declare that they have no competing interests.

\section{References}

1. Huang $\mathrm{H}$ and Tu L: Expression of S100 family proteins in neonatal rats with sepsis and its significance. Int J Clin Exp Pathol 8: 1631-1639, 2015.

2. Shane AL, Sánchez PJ and Stoll BJ: Neonatal sepsis. Lancet 390: 1770-1780, 2017.

3. Aydemir C, Aydemir H, Kokturk F, Kulah C and Mungan AG: The cut-off levels of procalcitonin and $\mathrm{C}$-reactive protein and the kinetics of mean platelet volume in preterm neonates with sepsis. BMC Pediatr 18: 253, 2018.

4. Giannoni E, Agyeman PKA, Stocker M, Posfay-Barbe KM, Heininger U, Spycher BD, Bernhard-Stirnemann S, Niederer-Loher A, Kahlert CR, Donas A, et al; Swiss Pediatric Sepsis Study: Neonatal sepsis of early onset, and hospital-acquired and community-acquired late onset: a prospective population-based cohort study. J Pediatr 201: 106-114.e4, 2018.

5. Tzialla C, Manzoni P, Achille C, Bollani L, Stronati M and Borghesi A: New diagnostic possibilities for neonatal sepsis. Am J Perinatol 35: 575-577, 2018. 
6. Wynn JL: Defining neonatal sepsis. Curr Opin Pediatr 28: 135-140, 2016.

7. Sharma D, Farahbakhsh N, Shastri S and Sharma P: Biomarkers for diagnosis of neonatal sepsis: a literature review. J Matern Fetal Neonatal Med 31: 1646-1659, 2018.

8. Memar MY, Alizadeh N, Varshochi M and Kafil HS Immunologic biomarkers for diagnostic of early-onset neonatal sepsis. J Matern Fetal Neonatal Med 32: 143-153, 2019.

9. Vishnoi A and Rani S: miRNA biogenesis and regulation of diseases: an overview. Methods Mol Biol 1509: 1-10, 2017.

10. Mishra S, Yadav T and Rani V: Exploring miRNA based approaches in cancer diagnostics and therapeutics. Crit Rev Oncol Hematol 98: 12-23, 2016.

11. Wang $X$, Wang $X$, Liu X, Wang $X, X u$ J, Hou S, Zhang $X$ and Ding Y: miR-15a/16 are upreuglated in the serum of neonatal sepsis patients and inhibit the LPS-induced inflammatory pathway. Int J Clin Exp Med 8: 5683-5690, 2015.

12. Huang HC, Yu HR, Huang LT, Huang HC, Chen RF, Lin IC, Ou CY, Hsu TY and Yang KD: miRNA-125b regulates TNFproduction in CD14+ neonatal monocytes via post-transcriptional regulation. J Leukoc Biol 92: 171-182, 2012.

13. Huang HC, Yu HR, Hsu TY, Chen IL, Huang HC, Chang JC and Yang KD: MicroRNA-142-3p and let-7g negatively regulates augmented IL-6 production in neonatal polymorphonuclear leukocytes. Int J Biol Sci 13: 690-700, 2017.

14. Chen J, Jiang S, Cao Y and Yang Y: Altered miRNAs expression profiles and modulation of immune response genes and proteins during neonatal sepsis. J Clin Immunol 34: 340-348, 2014.

15. Ahmad SF, Ansari MA, Nadeem A, Bakheet SA, Al-Ayadhi LY and Attia SM: Elevated IL-16 expression is associated with development of immune dysfunction in children with autism. Psychopharmacology (Berl) 236: 831-838, 2019.

16. Mathy NL, Scheuer W,Lanzendörfer M, Honold K, Ambrosius D, Norley S and Kurth R: Interleukin-16 stimulates the expression and production of pro-inflammatory cytokines by human monocytes. Immunology 100: 63-69, 2000.

17. Zhang F, Yang C, Xing Z, Liu P, Zhang B, Ma X, Huang L and Zhuang L: LncRNA GAS5-mediated miR-1323 promotes tumor progression by targeting TP53INP1 in hepatocellular carcinoma. OncoTargets Ther 12: 4013-4023, 2019.

18. Smith S, Wu PW, Seo JJ, Fernando T, Jin M, Contreras J, Montano EN, Gabhann JN, Cunningham K, Widaa A, et al: IL-16/miR-125a axis controls neutrophil recruitment in pristane-induced lung inflammation. JCI Insight 3: 120798, 2018

19. Plotnikov EY, Brezgunova AA, Pevzner IB, Zorova LD, Manskikh VN, Popkov VA, Silachev DN and Zorov DB Mechanisms of LPS-induced acute kidney injury in neonatal and adult rats. Antioxidants 7: E105, 2018.

20. Subspecialty Group of Neonatology Pediatric Society Chinese Medical Association; Editorial Board Chinese Journal of Pediatrics: Protocol for diagnosis and treatment of neonatal septicemia. Zhonghua Er Ke Za Zhi 41: 897-899, 2003 (In Chinese)

21. Yu HR, Chen RF, Hong KC, Bong CN, Lee WI, Kuo HC and Yang KD: IL-12-independent Th1 polarization in human mononuclear cells infected with varicella-zoster virus. Eur J Immunol 35: 3664-3672, 2005.

22. Livak KJ and Schmittgen TD: Analysis of relative gene expression data using real-time quantitative PCR and the 2(-Delta Delta C(T)) Method. Methods 25: 402-408, 2001

23. Rupaimoole R and Slack FJ: MicroRNA therapeutics: Towards a new era for the management of cancer and other diseases. Nat Rev Drug Discov 16: 203-222, 2017.

24. Ferrante $M$ and Conti GO: Environment and neurodegenerative diseases: an update on miRNA role. MicroRNA 6: 157-165, 2017

25. Correia de Sousa M, Gjorgjieva M, Dolicka D, Sobolewski C and Foti M: Deciphering miRNAs' action through miRNA editing. Int J Mol Sci 20: E6249, 2019.
26. Jeffries J, Zhou W, Hsu AY and Deng Q: miRNA-223 at the crossroads of inflammation and cancer. Cancer Lett 451: 136-141, 2019.

27. Yu A, Zhang T, Zhong W, Duan H, Wang S, Ye P, Wang J, Zhong S and Yang Z: miRNA-144 induces microglial autophagy and inflammation following intracerebral hemorrhage. Immunol Lett 182: 18-23, 2017.

28. Li Y, Ke J, Peng C, Wu F and Song Y: MicroRNA-300/NAMPT regulates inflammatory responses through activation of AMPK/mTOR signaling pathway in neonatal sepsis. Biomed Pharmacother 108: 271-279, 2018.

29. Cheng Q, Tang L and Wang Y: Regulatory role of miRNA-26a in neonatal sepsis. Exp Ther Med 16: 4836-4842, 2018.

30. Liu G, Liu W and Guo J: Clinical significance of miR-181a in patients with neonatal sepsis and its regulatory role in the lipopolysaccharide-induced inflammatory response. Exp Ther Med 19: 1977-1983, 2020.

31. Guo S, Mao X and Liang M: The moderate predictive value of serial serum CRP and PCT levels for the prognosis of hospitalized community-acquired pneumonia. Respir Res 19: 193, 2018.

32. Kim HS, Won S, Lee EK, Chun YH, Yoon JS, Kim HH and Kim JT: Pentraxin 3 as a clinical marker in children with lower respiratory tract infection. Pediatr Pulmonol 51: 42-48, 2016.

33. To KK, Tong CW, Wu M and Cho WC: MicroRNAs in the prognosis and therapy of colorectal cancer: From bench to bedside. World J Gastroenterol 24: 2949-2973, 2018.

34. Benz F, Roy S, Trautwein C, Roderburg C and Luedde T: Circulating microRNAs as biomarkers for sepsis. Int J Mol Sci 17: E78, 2016.

35. Ng PC, Chan KYY, Yuen TP, Sit T, Lam HS, Leung KT, Wong RPO, Chan LCN, Pang YLI, Cheung HM, et al: Plasma miR-1290 is a novel and specific biomarker for early diagnosis of necrotizing enterocolitis-biomarker discovery with prospective cohort evaluation. J Pediatr 205: 83-90.e10, 2019.

36. van Maldeghem I, Nusman CM and Visser DH: Soluble CD14 subtype (sCD14-ST) as biomarker in neonatal early-onset sepsis and late-onset sepsis: A systematic review and meta-analysis. BMC Immunol 20: 17, 2019.

37. Fatmi A, Rebiahi SA, Chabni N, Zerrouki H, Azzaoui H, Elhabiri Y, Benmansour S, Ibáñez-Cabellos JS, Smahi MC, Aribi M, et al: miRNA-23b as a biomarker of culture-positive neonatal sepsis. Mol Med 26: 94, 2020. Erratum in: Mol Med 26: $129,2020$.

38. Schüller SS, Wisgrill L, Herndl E, Spittler A, Förster-Waldl E, Sadeghi K, Kramer BW and Berger A: Pentoxifylline modulates LPS-induced hyperinflammation in monocytes of preterm infants in vitro. Pediatr Res 82: 215-225, 2017.

39. Zhang W, Lu F, Xie Y, Lin Y, Zhao T, Tao S, Lai Z, Wei N, Yang R, Shao $\mathrm{Y}$, et al: miR-23b negatively regulates sepsis-induced inflammatory responses by targeting ADAM10 in human THP-1 monocytes. Mediators Inflamm 2019: 5306541, 2019.

40. Roth S, Solbach W and Laskay T: IL-16 and MIF: Messengers beyond neutrophil cell death. Cell Death Dis 7: e2049, 2016.

41. Sugitharini V, Prema A and Berla Thangam E: Inflammatory mediators of systemic inflammation in neonatal sepsis. Inflamm Res 62: 1025-1034, 2013

42. Huang Y, Du KL, Guo PY, Zhao RM, Wang B, Zhao XL and Zhang CQ: IL-16 regulates macrophage polarization as a target gene of mir-145-3p. Mol Immunol 107: 1-9, 2019.

43. Xia D, Yao R, Zhou P, Wang C, Xia Y and Xu S: LncRNA NEAT1 reversed the hindering effects of miR-495-3p/STAT3 axis and miR-211/PI3K/AKT axis on sepsis-relevant inflammation. Mol Immunol 117: 168-179, 2020.

This work is licensed under a Creative Commons Attribution-NonCommercial-NoDerivatives 4.0 International (CC BY-NC-ND 4.0) License. 